\title{
Erratum to: A novel infrared laser device that measures multilateral parameters of stepping performance for assessment of fall risk in elderly individuals
}

\author{
Shu Nishiguchi $\cdot$ Minoru Yamada $\cdot$ Kazuki Uemura $\cdot$ \\ Tetsuya Matsumura • Masaki Takahashi • \\ Toshiki Moriguchi $\cdot$ Tomoki Aoyama
}

Published online: 1 June 2013

(C) Springer International Publishing Switzerland 2013

\section{Erratum to: Aging Clin Exp Res}

DOI 10.1007/s40520-013-0042-9

There was an error in the title.

The correct title is:

A novel infrared laser device that measures multilateral parameters of stepping performance for assessment of fall risk in elderly individuals

The online version of the original article can be found under doi:10.1007/s40520-013-0042-9.

S. Nishiguchi $(\bowtie) \cdot$ M. Yamada $\cdot$ T. Aoyama

Department of Physical Therapy, Human Health Sciences,

Graduate School of Medicine, Kyoto University,

53 Kawahara-cho, Shogoin, Sakyo-ku, Kyoto 606-8507, Japan

e-mail: nishiguchi.shu.82s@st.kyoto-u.ac.jp

\section{K. Uemura}

Department of Physical Therapy, Graduate School of Medicine,

Nagoya University, 1-1-20 Daikouminami, Higashi-ku,

Nagoya 461-8673, Japan

T. Matsumura

Graduate School of Science and Technology, Keio University,

3-14-1 Hiyoshi, Kohoku-ku, Yokohama,

Kanagawa 223-8522, Japan

M. Takahashi

Department of System Design Engineering, Keio University,

3-14-1 Hiyoshi, Kohoku-ku, Yokohama,

Kanagawa 223-8522, Japan

T. Moriguchi

Research and Development Division, Murata Machinery, Ltd.,

136 Takeda-Mukaishiro-cho, Fushimi-ku,

Kyoto 612-8686, Japan 